\title{
Analysis of Emotion and Engagement in a STEM Alternate Reality Game
}

\author{
Yu-Han Chang, Rajiv Maheswaran, Jihie Kim, and Linwei Zhu \\ University of Southern California \\ Information Sciences Institute \\ Marina del Rey, CA 90292 \\ \{ychang, maheswar, jihie\}@isi.edu, vic90228@gmail.com
}

\begin{abstract}
Alternate reality games (ARGs) are a promising new approach for increasing student engagement; however, automated methods for analyzing and optimizing game play are non-existent. We captured the player communication generated by a recent STEM-focused ARG that we piloted in a Los Angeles charter high school. We used shallow sentiment analysis to gauge the levels of various emotions experienced by the players during the course of the game. Pre/post-game surveys gauged whether the game narratives had any effect on student engagement and interest in STEM topics.
\end{abstract}

\section{Introduction}

Alternate Reality Games (ARGs) are a relatively new genre that has shown promise for engaging students in STEM learning activities. These transmedia experiences typically draw participants into fictional narratives, where players interact via various forms of social and traditional media, and frequently become part of the storyline themselves. They differ from traditional virtual reality computer games, where the entire story takes place in a fictional online world. In ARGs, the game world overlaps with the real world. Players visit real places, research the real world wide web, communicate with other players and fictional characters using real social media, phone, text messaging, and occasionally live encounters in the real world. For education, this novel game format has the potential to literally bring science activities and learning into the normal lives of students, emphasizing STEM relevance to the students context, surroundings, and community. The ARG brings the game space into the physical daily reality of students [?,?].

In this paper, we describe a pilot ARG we designed and implemented at USC Hybrid High in Fall 2012. We describe the ways in which we were able to capture player data, both by observing the players in game, and by validating these observations through pre and post game tests. In order for ARGs to truly support educational objectives, we need to be able to unobtrusively measure and understand the performance of players within the game, using only their in-game, visible interactions, such as website visitation and forum postings. Individual 
player assessment enables puppetmasters to tweak the game play to maximize engagement and educational outcome for each learner. Clearly AI and other computational techniques are needed to reach this goal, and this short paper only presents a summary of a small step in this direction.
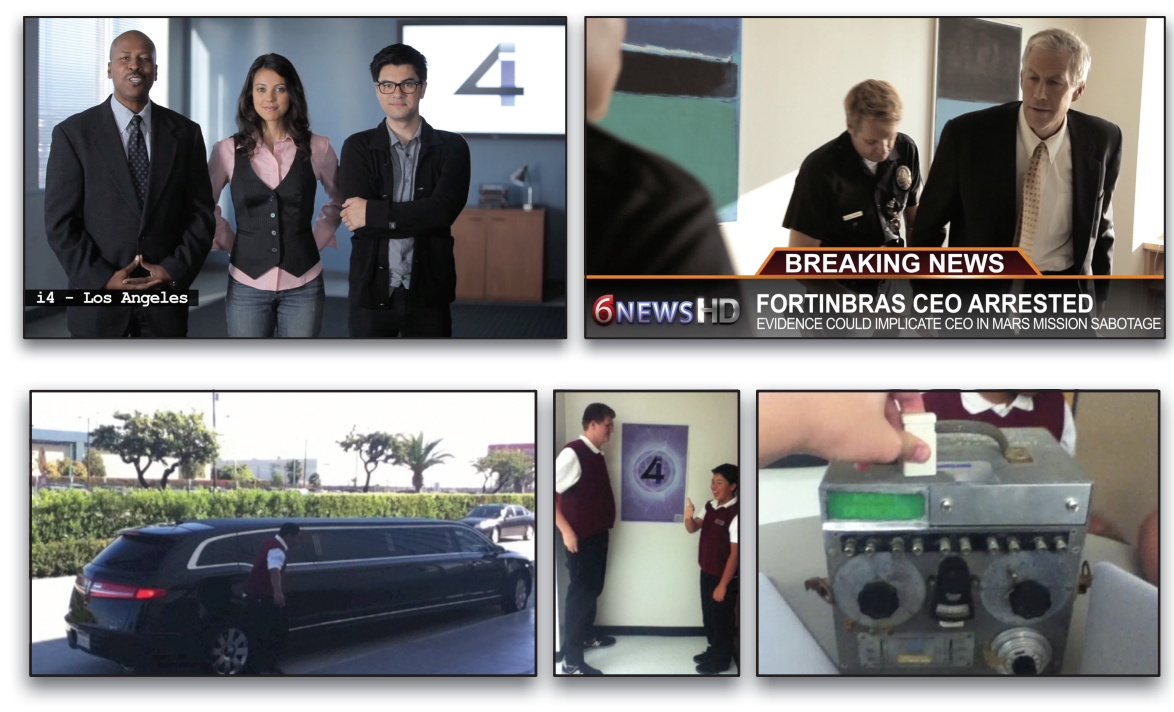

Fig. 1. (Top Left) The main characters in the game: William, Isa, and Rudy, (T. Right) The final story element in the game, where Fortinbras' CEO is arrested. (Bottom Left) Special trip to Space X facilities, (B. Center) Mysterious poster at USC Hybrid High, (B. Right) Device used to thwart Fortinbras.

USC Hybrid High ARG Pilot: Operation Daylight. In Fall 2012, we fielded a pilot alternate reality game, "Operation Daylight," at USC Hybrid High, a new charter high school with approximately 100 ninth graders in its inaugural class. The population is almost entirely minority and receive free/reduced lunches. The game focuses on $\pi$, an organization set up centuries ago to defend science. Its most recent incarnation, i4, needs students from USC Hybrid High to be their next generation, and the game begins with i4 recruiting and training students from the school. In the process, the students complete STEM-related activities to advance up the i4 recruitment ladder.

Gradually, the students uncover an evil plot by Fortinbras Industries that threatens their protagonist recruitment agents, the fictional characters Rudy Vanzant and Isa Figueroa, played by local actors in a variety of video sequences. This requires the students to put their newly learned skills to real use in order to save their friends Rudy and Isa. Figure ?? shows some of the elements used in the game. The game ran for approximately five weeks at USC Hybrid High, from $10 / 18 / 12$ to $11 / 21 / 12$. It was a completely optional activity that students 
could engage in if they chose to, with both online, at-school, and out-of-school elements. Students drove over 27,670 page views to the i4 website and posted 1394 messages to the i4 forum.

\section{Methodology and Results}

We used well-established scales for measuring student interest in STEM topics developed by OECD's Programme for International Student Assessment (PISA) [?]. Pre and post game surveys were developed using these scales, and administered to students at USC Hybrid High one week before the game commenced and one week after the game concluded. The surveys included approximately thirty questions where students would respond "Strongly Agree", "Agree", "Disagree", or "Strongly Disagree." The survey also included questions that established basic demographic information, as well as self-reported aspects of game play. In addition to the survey data, we also collected in-game data such as forum visits, messages posted, videos and pictures posted. We also used the Linguistic Inquiry and Word Count (LIWC) text analysis tool to process the messages [?] and detect whether they expressed a positive or negative sentiment, or whether the message contained anxiety, fear, or happiness.

Fifty-nine out of the 94 survey respondents indicated that they had heard of i4 and the Operation Daylight game. Twenty-three of the 29 students who signed up on the Operation Daylight website filled out surveys. Among students who played the game, they overwhelmingly thought the game increased their interest in science $(48 \%)$ or did not change their already positive interest in science $(47 \%)$. No one ended up having less interest in science.

These responses are corroborated with the students' answers to the OECD science interest questions. Figure ?? shows how the students' science interest levels changed from the beginning of the game to the end of the game, conditioned how often they visited the i4 forum, and on the average length of their posts on the forum. In these graphs, 0 corresponds to "Strongly disagree" (dislike science), and 3 corresponds to "Strongly agree" (like science). We see that there is a correlation between more visits and higher science interest level, as well as between longer posts and higher interest levels. There also appears to be a correlation between longer posts and a larger amount of increase in science interest.

Figure ?? shows that there is a correlation between forum activity and the major game events, such as the main characters being abducted. This suggests that these ARG story elements might promote the higher science interest levels described above. We also analyze the number of messages that contained certain percentages of message words that indicate positive or negative attitude, anxiety, fear, or sadness. It turned out that there is no clear pattern between the story elements and the production of particular categories of words, contrary to our expectation. For example, the abduction of the main character did not obviously produce more messages of fear or negativity. Generally the proportional levels of positive words stays constant during the game, and the levels of negative words 

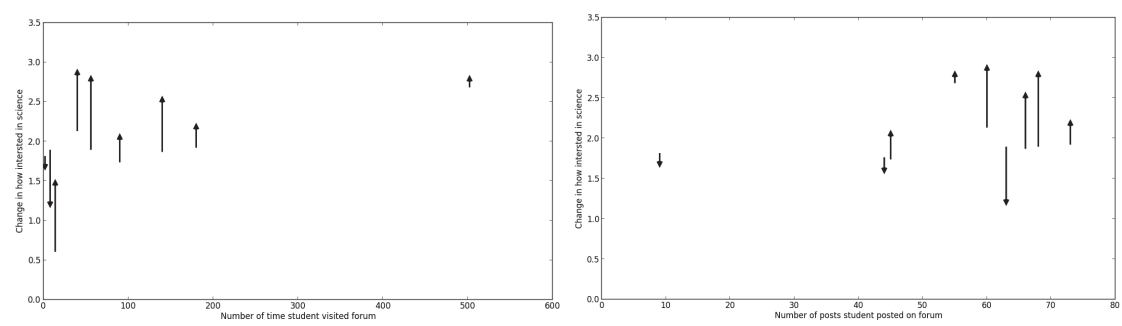

Fig. 2. (Left) Number of visits vs. change in science interest levels, (Right) Average length of forum postings vs. change in science interest levels.

stays quite low. The proportions of messages with varying levels positive words are also shown in Figure ??. Due to lack of space here, a longer version of this paper will be posted at our website, http://cb.isi.edu.

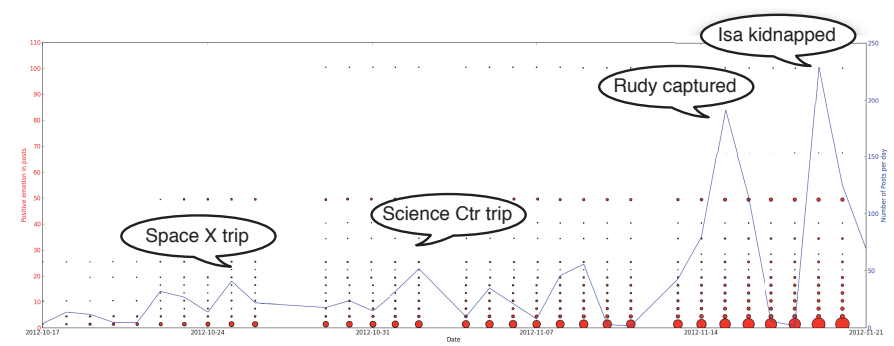

Fig. 3. Time showing the level of forum activity over the course of the game. The thin blue line denotes the number of posts in the forum on each day, the red circles denote how many of those messages contained a particular fraction of positive words.

Acknowledgements. This work was supported by NSF Award \#:1224088.

\section{References}

1. E. Klopfer. Augmented learning: Research and design of mobile educational games. MIT Press, 2008.

2. Herbert W. Marsh, Kit-Tai Hau, Artelt Cordula, Baumert Jurgen, and Jules L. Peschar. Oecd's brief self-report measure of educational psychology's most useful affective constructs: Cross-cultural psychometric comparisons across 25 countries. International Journal of Testing, 6(4):311 360, 2006.

3. A. Moseley, N. Whitton, J. Culver, and K. Piatt. Motivation in alternate reality gaming environments and implications for learning. In 3rd European Conference on Games Based Learning. Academic Conferences Limited, 2009.

4. J.W. Pennebaker. Putting stress into words: Health, linguistic, and therapeutic implications. Behaviour research and therapy, 31(6):539-548, 1993. 